\title{
Impulsive Softening of Coherent Phonons in Tellurium
}

\author{
S. Hunsche,* K. Wienecke, T. Dekorsy, and H. Kurz \\ Institut für Halbleitertechnik II, Rheinisch Westfälische Technische Hochschule Aachen, 52056 Aachen, Germany
}

\begin{abstract}
We investigate the dynamics of coherent optical phonons in tellurium after high-density excitation with femtosecond laser pulses. The data show a continuous redshift of the phonon frequency with increasing excitation density. Experiments with double-pulse excitation prove that the observed frequency shift is of purely electronic origin. We demonstrate that coherent phonons allow monitoring the pathway to nonthermal laser-induced melting of crystalline materials.
\end{abstract}

Since the optical excitation of semiconductor crystals corresponds to a promotion of electrons from bonding into antibonding states, high-density excitation may lead to a nonthermal melting, without heating of the lattice to the melting temperature [1]. The occurrence of laser-induced phase transitions has been experimentally demonstrated in time-resolved studies of second-harmonic generation on $\mathrm{Si}$ and GaAs [2,3]. In these experiments, a loss of the crystal structure was observed on a femtosecond time scale, i.e., before significant energy transfer between the excited electrons and the lattice can occur. However, these experiments are sensitive to the crystal symmetry and can only indicate whether the phase transition has taken place or not. In this Letter, we present a study of coherent phonons in highly excited tellurium, where we monitor the weakening of the crystal via the reduction of the phonon frequency. We show that this shift is of almost purely electronic origin and directly related to the electron density. Therefore our experiments provide direct information about the pathway towards a laserinduced phase transition.

Optical excitation and time-domain detection of coherent lattice vibrations require femtosecond laser pulses with a duration much shorter than the vibration period and has been demonstrated in various materials [4-7]. One of the most efficient generation mechanisms is the "displacive excitation of coherent phonons" (DECP) that has been identified in experiments with several materials, including tellurium [5]. This mechanism results from the fact that the lattice equilibrium position of an electronically excited state differs from that of the ground state. Therefore above-band-gap optical excitation with an ultrashort laser pulse prepares the lattice in a state displaced from its new equilibrium position, leading to coherent lattice vibrations with a characteristic cosinelike time dependence [8].

Up to now, most studies of coherent phonons have been performed in a low-excitation regime, so that the coherent phonon frequencies match exactly the frequencies observed in nonresonant Raman measurements. Only in two recent studies has a transient shift of the phonon frequency been reported, which has been assigned to the large ionic displacement [9], i.e., anharmonicity of the phonon, and to "ionic screening" by the photoexcited carriers [10], where the time dependence has been interpreted in terms of screening efficiency. However, recent theoretical investigations predict a continuous weakening of the lattice with increasing electron density and a complete instability when $\approx 9$ of all valence electrons in a semiconductor are excited [11]. Clearly, in this extreme case the restoring forces for transverse lattice displacements vanish so that the transverse phonon frequencies become zero.

Our experiments are performed in a standard femtosecond pump-probe scheme. Femtosecond pulses of a commercial Ti-sapphire oscillator are amplified to a pulse energy of $1.2 \mu \mathrm{J}$ in a six-pass dye (Styryl $9 \mathrm{~m}$ ) laser amplifier pumped by a $6.8 \mathrm{kHz}$ copper vapor laser. Compensation of the amplifier dispersion by a four-prism sequence results in pulse durations below $100 \mathrm{fs}$. In one series of measurements, the pump pulses are split into two, one of which is used as a prepump that creates a certain carrier density at a negative time delay. In all experiments, we keep the pump foci relatively large $(70-100 \mu \mathrm{m})$ and the probe spot about a factor of 2 smaller to reduce the influence of lateral inhomogeneity [12]. Pump-induced reflectivity changes are measured with a lock-in amplifier as a function of time delay between pump and probe, which is controlled with a stepper motor.

The sample is single-crystal tellurium, with the surface oriented perpendicular to the $c$ axis. The DECP mechanism leads to the excitation of the highly symmetric nonpolar $A_{1}$ mode, for which a frequency of $3.6 \mathrm{THz}$ is found in conventional Raman measurements at room temperature [13].

We estimate the photoexcited carrier density from the maximum fluence per pulse (in the center of the excitation spot). Assuming strictly linear absorption, as described by the absorption coefficient of $2 \times 10^{5} \mathrm{~cm}^{-1}$ at $825 \mathrm{~nm}$, a maximum fluence of, e.g., $2.4 \mathrm{~mJ} / \mathrm{cm}^{2}$ will give a density of $1.1 \times 10^{21} \mathrm{~cm}^{-3}$ at the sample surface, which is about $2 \%$ of all valence electrons.

The extremely small absorption length of only $50 \mathrm{~nm}$ will lead to enormous density gradients at high pump intensities, so that carrier diffusion into the crystal becomes 
important even on a subpicosecond time scale. As a measure for the typical time scale, the time needed for a carrier to diffuse over one absorption length can be easily estimated from the equilibrium ambipolar diffusion constant of $40 \mathrm{~cm}^{2} / \mathrm{s}$ [13]. This time will be about $600 \mathrm{fs}$ for a maximum excitation density of $1.1 \times 10^{21} \mathrm{~cm}^{-3}$.

Figure 1 shows typical pump-probe data after singlepulse excitation with varying excitation density. The general time dependence of the reflectivity changes can be described by an instantaneous increase during the pump pulse duration and subsequent damped oscillations superimposed on a slowly decaying background [5]. This background can be assigned to direct changes of the electronic susceptibility by the photoexcited carriers. In contrast, the oscillations stem from the impulsively excited vibration of the $A_{1}$ phonon that leads to an isotropic modulation of refractive index via the diagonal elements of the Raman tensor. Other phonon modes are not detected in our measurements, as they are generated less effectively and cause much smaller reflectivity changes [7].

The data in Fig. 1 clearly show that the amplitudes of both the background signal and the oscillations increase monotonically with the pump fluence. For fluences below $\approx 3 \mathrm{~mJ} / \mathrm{cm}^{2}$, both signals increase linearly with the excitation power, while we find a saturation for higher fluences. Therefore the uppermost curve in Fig. 1 has been multiplied by a factor of 1.5 for better visibility. Based on the linear increase of the background amplitude with excitation power, this background may be taken as a direct measure of the photoexcited carrier density. This interpretation is consistent with the faster decay of the background at higher densities, which can be attributed to the carrier diffusion into the crystal.

On the other hand, the linear increase of the oscillation amplitude with excitation fluence is a direct consequence of the DECP mechanism that launches the coherent phonons. In addition to this linearity, all our measurements confirm the predicted cosine time depen-

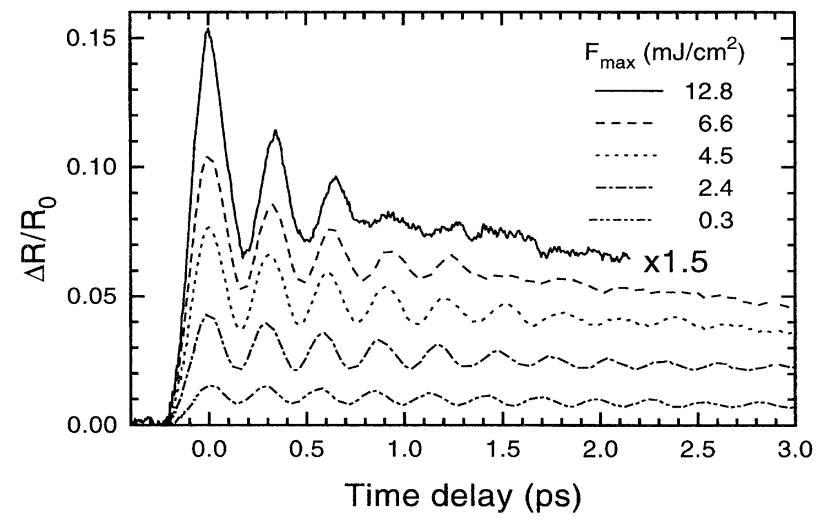

FIG. 1. Time-resolved reflectivity changes at different excitation fluences. The first oscillation maximum is arbitrarily shifted to time delay zero. dence of the oscillations [8]. The data in Fig. 1 also suggest that the coherent phonons decay faster at high excitation density. While the dephasing time is about 2 ps at the lowest density, as determined from fitting the data, the oscillations vanish completely within $1 \mathrm{ps}$ at $F=12 \mathrm{~mJ} / \mathrm{cm}^{2}$. More striking is the fact that the oscillation period shows a continuous increase, i.e., the phonon frequency decreases, with increasing excitation density. At the highest pump fluences the oscillation period also change slightly with time delay.

The redshift of the phonon frequency is illustrated by the Fourier spectra of the oscillating signal contributions shown in Fig. 2. These spectra are calculated after fitting and subtracting the slowly varying background signal. Clearly, the maximum frequency of the phonon response drops from 3.6 down to $3 \mathrm{THz}$ at the highest fluence. In addition, the spectra show a strongly asymmetric broadening at high fluences. While the phonon response is symmetrically distributed around its maximum at low excitation density, it shows a significant low-frequency tail that extends well below $1 \mathrm{THz}$ at the highest density. The decrease of the maximum intensity for $F>3 \mathrm{~mJ} / \mathrm{cm}^{2}$ corresponds to the saturation of the amplitude in combination with the increasing damping of the coherent phonons.

The dependence of the phonon frequency on the pump fluence is depicted in Fig. 3. The solid dots give the maximum of the Fourier transforms, while the open symbols show the instantaneous frequency fitted for single cycles of the oscillations. For fluences below $\approx 6 \mathrm{~mJ} / \mathrm{cm}^{2}$, there is no systematic deviation between these different values, i.e., both may be interpreted as the actual phonon frequency. Obviously, the density dependence of the phonon frequency can be well fitted by a linear decrease from the literature value at $F_{\max }=0$. For high intensities, there is some relaxation of the singlecycle frequencies towards higher values, but the inverse period of the first oscillation still shows a linear decrease and lies well below the Fourier maximum. We note that the relaxation of the oscillation frequency corresponds to a

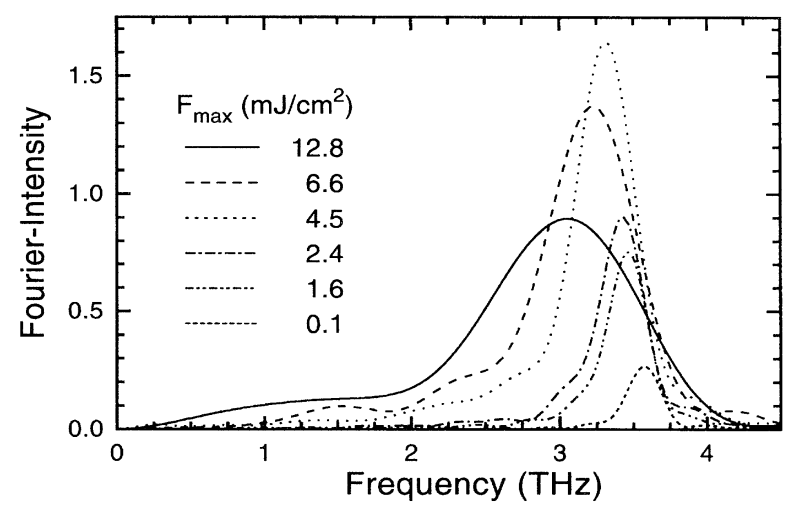

FIG. 2. Fourier transforms of the oscillating signal contribution at various pump fluences. 


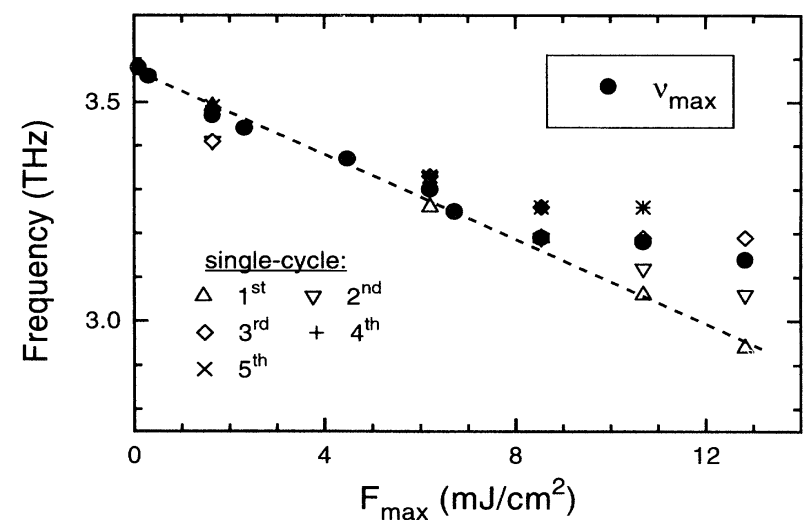

FIG. 3. Excitation-density dependence of the phonon frequency. Solid circles show the maximum of the Fourier transforms, open symbols the "instantameous" frequencies determined by fitting different cycles of the oscillating signal.

fast relaxation of the electronic background signal at high pump intensities.

Taking into account the inhomogeneity of the pumpcreated carrier density (see below), the data indicate a linear decrease of the phonon frequency with carrier density. The estimation of the photoexcited carrier density achieved in our experiments shows that several percent of the valence electrons are excited at the highest pump intensities, so that a considerable weakening of the crystal is expected [11]. Therefore the observed reduction of the restoring force for the $A_{1}$ lattice displacement appears as a natural consequence of the excitation of a large number of electrons from bonding into antibonding states. This effect may be described as a purely electronic softening of the crystal lattice.

In a recent report, Cheng et al. have observed a transient redshift of the phonon frequency in $\mathrm{Sb}$ and assigned this to ionic screening by the photoexcited carriers [10]. However, screening effects can be excluded for the case of tellurium, since the crystal bonds are predominantly covalent rather than ionic. Also, there is no plasmon-phonon coupling [12] for the $A_{1}$ phonon, since this is not infrared active. An additional softening due to lattice heating or emission of nonequlibrium phonons by the photoexcited carriers - which is expected to take place on a ps time scale - appears to be negligible in our experiments, since the frequency shifts occur "instantaneously" with the excitation. In addition, seperate experiments showed that the influence of lattice heating on the coherent phonon frequency is much smaller than that of the photoexcitation [14].

In order to understand the shape of the phonon response and the partial relaxation of the oscillation frequency, the strong inhomogeneity of the initial carrier density has to be taken into account. The probe pulse "sees" signal contributions from different depths below the sample surface, corresponding to different carrier densities. Increasing the excitation fluence corresponds to an increasing range of densities, so that a density-dependent redshift will lead to an asymmetric broadening of the phonon response, as observed in Fig. 2, and to an apparent decrease of the coherent phonon dephasing time. Therefore the distinct low-frequency tail of the phonon response at the highest pump intensities suggests a substantial mode softening in the region of highest carrier density that would be underestimated if only the shift of the maximum were taken into account.

On the other hand, the large density gradient associated with the short absorption length will lead to a very fast carrier diffusion at high excitation power and therefore to a rapid decrease of the carrier density at the surface. Assuming a monotonous decrease of the frequency with carrier density provides a consistent explanation for the temporal shift of the instantaneous oscillation frequency, which corresponds directly to the rapid decay of the electronic background signal.

To check the consistency of our interpretation and to prove the electronic origin of the frequency shifts, we have performed additional experiments with doublepulse excitation of the sample: We use a prepump with variable intensity to create a certain carrier density and observe the influence of these carriers on the dynamics of coherent phonons generated by a second pump pulse. To avoid interference effects of the coherent phonons [15], we chose a time delay of 6 ps between the pump pulses, so that the oscillations created by the prepump have completely vanished when the second pulse hits the sample.

In Fig. 4 we compare the phonon frequency shift under single- and double-pulse excitation. The experimental situation is illustrated in the inset, which shows the transient reflectivity changes induced by the double-pulse excitation. In the double-pump experiment, the fluence of the second pump is kept constant at $F=0.5 \mathrm{~mJ} / \mathrm{cm}^{2}$, while the fluence of the prepump is varied from 0.3 to $1.5 \mathrm{~mJ} / \mathrm{cm}^{2}$. For this density range, we did not find a delay dependence of the frequency. Therefore in Fig. 4 we show a single frequency for each measurement, which is obtained by fitting the oscillating signal contribution for positive time delays. In the time interval between the two excitation pulses there is a significant decrease of the electronic background signal created by the prepump, due to carrier diffusion into the sample. To allow a direct comparison of the two types of experiments, we show the data as a function of the maximum electronic background at time delay zero, which is taken as a direct measure of the effective total carrier density created by both pumps. The data clearly show an identical dependence of the oscillation frequency in both types of experiments. As the phonons created by the second pump are affected only by varying the carrier density excited by the prepump, this is an unambiguous proof for a predominantly electronic origin of the observed 


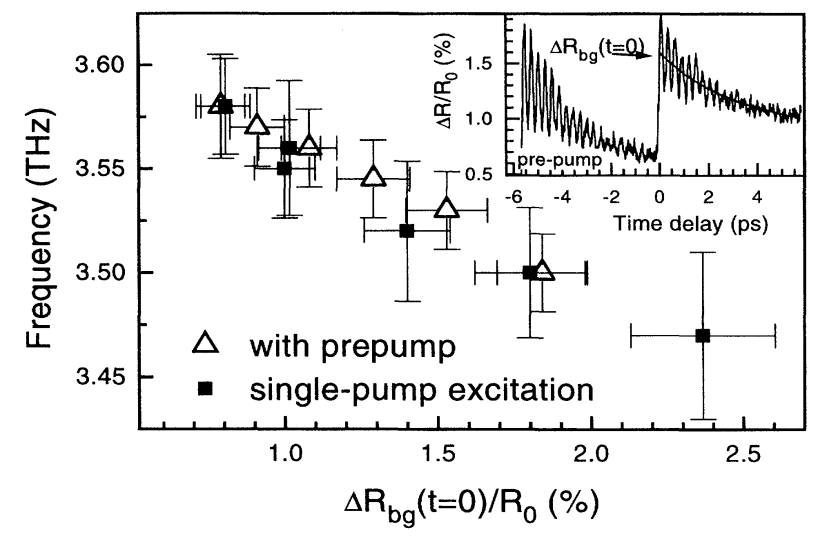

FIG. 4. Phonon frequency shift under single-pulse and double-pulse excitation. The electronic background signal at $t=0$ is used as a measure of the effective carrier density. The inset illustrates the double-pulse experiment.

mode softening. A possible influence of anharmonicity of the phonon itself $[9,16]$ can be safely excluded, since the intensity of the second pump, and thus the phonon amplitude, was kept constant.

Figure 5 shows the values of the coherent phonon dephasing time, as determined from fitting the data with a damped cosine, for single- and double-pulse excitation. In contrast with the frequency, the dephasing time remains quite unaffected by changing the intensity of the prepump. This indicates that there is no significant direct coupling between the phonon and the free carriers. Therefore the decrease of the dephasing times observed in the singlepump experiments can only be assigned to the increasing inhomogeneity, as already concluded from the shape of the Fourier spectra. In the double-pulse experiment the delay between the first and second pump is sufficiently large to allow a significant reduction of the spatial inhomogeneity by carrier diffusion. The slight offset of the dephasing times in the double-pulse experiment may be attributed to an increase of the lattice temperature due to the prepulse

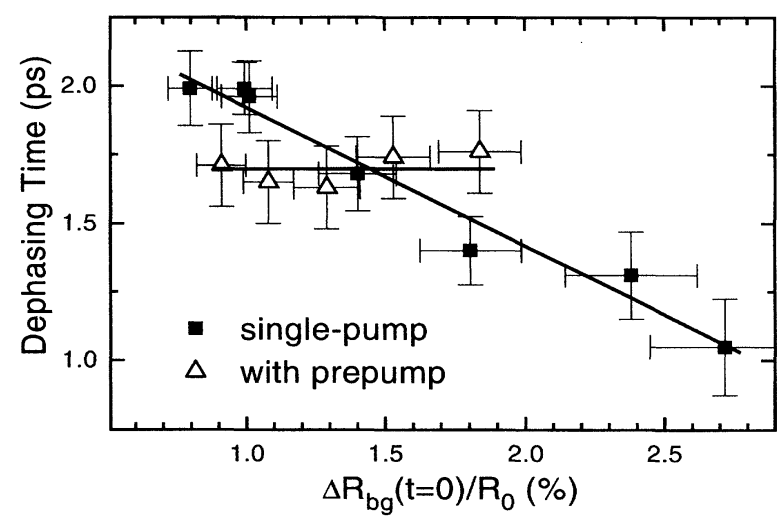

FIG. 5. Density dependence of the coherent phonon dephasing time under single-pulse and double-pulse excitation.
[14], however, this explanation is not crucial for the interpretation of the main results of this paper.

In conclusion, we present an experimental investigation of the coherent phonon dynamics in tellurium as a function of excitation density. All experimental results can be consistently explained in terms of a monotonous decrease of the $A_{1}$-phonon frequency with increasing carrier density, i.e., an electronic weakening of the crystal lattice. For the highest pump powers studied, the photoexcited carrier densities can be estimated to be several percent of all valence electrons. At these densities the phonon response contains low-frequency contributions well below $1 \mathrm{THz}$, suggesting a substantial destabilization of the crystal by the optical excitation. Therefore our experiments provide microscopic information that cannot be obtained from experimental methods that are only sensitive to changes of the crystal structure.

We are grateful to Professor P. Grosse for supplying the samples. This work has been supported by the Deutsche Forschungsgemeinschaft.

*Present address: FOM-Institute for Atomic and Molecular Physics, Kruislaan 407, 1098 SJ Amsterdam, The Netherlands

[1] J. A. Van Vechten, R. Tsu, and F. W. Saris, Phys. Lett. 74A, 422 (1979).

[2] C. V. Shank, R. Yen, and C. Hirlimann, Phys. Rev. Lett. 51, 900 (1983); H. W. K. Tom, G. D. Aumiller, and C. H. Brito-Cruz, ibid. 60, 1438 (1988).

[3] P. Saeta et al., Phys. Rev. Lett. 67, 1023 (1991); S. V. Govorkov et al., Optics Lett. 16, 1013 (1991); K. Sokolowski-Tinten et al., Appl. Phys. A 53, 227 (1991); Phys. Rev. B 51, 14186 (1995).

[4] S. DeSilvestri et al., Chem. Phys. Lett. 116, 146 (1985).

[5] T. K. Cheng et al., Appl. Phys. Lett. 57, 1004 (1990); T. K. Cheng et al., ibid. 59, 1923 (1991).

[6] G. C. Cho, W. Kütt, and H. Kurz, Phys. Rev. Lett. 65, 764 (1990); W. Kütt, W. Albrecht, and H. Kurz, IEEE J. Quantum. Electron. 28, 2434 (1992);

[7] T. Dekorsy et al., Phys. Rev. Lett. 74, 738 (1995).

[8] H. J. Zeiger et al., Phys. Rev. B 45, 768 (1992).

[9] T. K. Cheng, M.S. Dresselhaus, and E.P. Ippen, Appl. Phys. Lett. 62, 1901 (1993).

[10] T. K. Cheng et al., Ultrafast Phenomena IX, edited by P.F. Barbara and W.H. Knox, Springer Series in Chemical Physics, Vol. 60 (Springer, Berlin, 1994), p. 301.

[11] P. Stampfli and K. H. Bennemann, Phys. Rev. B 42, 7163 (1990); 46, 10686 (1992); 49, 7299 (1994).

[12] A. V. Kutznetzov and C. J. Stanton, Phys. Rev. B 51, 7555 (1995).

[13] P. Grosse and W. Richter, in Landolt-Börnstein, New Series, edited by O. Madelung (Springer, Berlin, 1983), Vol. 17.

[14] K. Wienecke (unpublished).

[15] T. Dekorsy et al., Europhys. Lett. 23, 223 (1993).

[16] C. M. Foster et al., Phys. Rev. B 48, 10160 (1993); H. J. Bakker, S. Hunsche, and H. Kurz, ibid. 48, 13524 (1993); 50, 914 (1994). 